\title{
Effect of early supervised physiotherapy on recovery from acute ankle sprain: randomised controlled trial
}

\author{
Robert J Brison, ${ }^{1,2,3}$ Andrew G Day, ${ }^{2,3}$ Lucie Pelland, 4,5 William Pickett, ${ }^{1,3}$ Ana P Johnson, ${ }^{3}$ Alice Aiken, ${ }^{4}$ \\ David R Pichora, 5,6 Brenda Brouwer ${ }^{4,5}$
}

${ }^{1}$ Queen's University Department of Emergency Medicine,

Kingston, ON, Canada

${ }^{2}$ Kingston General Hospital Research Institute, Kingston, ON, Canada

${ }^{3}$ Queen's University Department of Public Health Sciences,

Kingston, ON, Canada

4Queen's University School of

Rehabilitation Therapy,

Kingston, ON, Canada

${ }^{5}$ The Human Mobility Research Centre, Kingston General

Hospital and Queen's

University, Kingston, ON,

Canada

'Queen's University Division of Orthopaedics, Kingston, ON

Canada

Correspondence to: R J Brison, Queen's University, Clinical

Research Centre, Victory 3,

Kingston General Hospital,

76 Stuart Street, Kingston,

ON, K7L 2V7, Canada

brisonr@kgh.kari.net

Cite this as: BMJ 2016;355:i5650 http://dx.doi.org/10.1136/bmj.i5650

Accepted: 18 October 2016

\author{
ABSTRACT \\ OBJECTIVE \\ To assess the efficacy of a programme of supervised \\ physiotherapy on the recovery of simple grade 1 and 2 \\ ankle sprains.
}

DESIGN

A randomised controlled trial of 503 participants followed for six months.

SETTING

Participants were recruited from two tertiary acute care settings in Kingston, ON, Canada.

\section{PARTICIPANTS}

The broad inclusion criteria were patients aged $\geq 16$ presenting for acute medical assessment and treatment of a simple grade 1 or 2 ankle sprain. Exclusions were patients with multiple injuries, other conditions limiting mobility, and ankle injuries that required immobilisation and those unable to accommodate the time intensive study protocol.

\section{INTERVENTION}

Participants received either usual care, consisting of written instructions regarding protection, rest, cryotherapy, compression, elevation, and graduated weight bearing activities, or usual care enhanced with a supervised programme of physiotherapy.

\section{MAIN OUTCOME MEASURES}

The primary outcome of efficacy was the proportion of participants reporting excellent recovery assessed with the foot and ankle outcome score (FAOS). Excellent recovery was defined as a score $\geq 450 / 500$ at three months. A difference of at least $15 \%$ increase in the absolute proportion of participants with excellent recovery was deemed clinically important. Secondary

\section{WHAT IS ALREADY KNOWN ON THIS TOPIC}

Ankle sprains are one of the most common and burdensome musculoskeletal injuries and are associated with a high rate of visits to an emergency department Although most ankle sprains are simple grade 1 or grade 2 ligament sprains, the clinical standards for the acute management of these injuries are not well defined Current evidence regarding the role of supervised physiotherapy in the acute management of these injuries is limited

\section{WHAT THIS STUDY ADDS}

In the acute management of patients with grade 1 and 2 ankle sprains presenting to an emergency department within 72 hours of injury, a standard intervention of early supervised physiotherapy plus usual care does not lead to clinically important improvements in functional recovery up to six months after injury compared with usual care alone

As $43 \%$ of participants in the physiotherapy arm and $38 \%$ in the control arm did not reach excellent recovery by six months, there is potential for the investigation of alternative interventions that would reduce morbidity associated with these injuries analyses included the assessment of excellent recovery at one and six months; change from baseline using continuous scores at one, three, and six months; and clinical and biomechanical measures of ankle function, assessed at one, three, and six months.

\section{RESULTS}

The absolute proportion of patients achieving excellent recovery at three months was not significantly different between the physiotherapy $(98 / 229,43 \%)$ and usual care $(79 / 214,37 \%)$ arms (absolute difference 6\%, 95\% confidence interval-3\% to $15 \%)$. The observed trend towards benefit with physiotherapy did not increase in the per protocol analysis and was in the opposite direction by six months. These trends remained similar and were never statistically or clinically important when the FAOS was analysed as a continuous change score.

\section{CONCLUSIONS}

In a general population of patients seeking hospital based acute care for simple ankle sprains, there is no evidence to support a clinically important improvement in outcome with the addition of supervised physiotherapy to usual care, as provided in this protocol.

TRIAL REGISTRATION

ISRCTN 74033088 (www.isrctn.com/ISRCTN74033088)

\section{Introduction}

Ankle sprains are one of the most common and burdensome musculoskeletal injuries and are associated with a high rate of visits to the emergency department. ${ }^{1-6}$ In our own setting of Kingston, Canada, ankle sprains account for $7.5 \%$ of injury related visits, about 725 cases annually, which represents an annual rate of presentation to an emergency department of five per 1000 people. Nearly all ankle sprains are simple grade 1 (mechanically stable) or grade 2 (some joint laxity) ligament sprains. Grade 3 sprains (clinical and/or radiological evidence of instability) represent a small minority. ${ }^{7-9}$ Although the prevalence of grade 3 sprains is low, there is good evidence to support the use of immobilisation and, occasionally, surgical correction in the management of these injuries. ${ }^{10-12}$ Yet clinical standards for the acute management of grade 1 and 2 ankle sprains are not well defined.

The absence of high quality evidence to inform effective management of ankle sprains in acute care settings is related largely to perceptions that grade 1 and 2 ankle sprains are relatively benign injuries. ${ }^{13}$ Short and long term morbidity from simple ankle sprains, however, has been documented among $30-70 \%$ of patients, ranging from six months to seven years after injury. ${ }^{10-121415}$ 
Morbidity is reflected in lost time from school, work, leisure, and sports activities ${ }^{15-17}$; impairment in activities of daily living ${ }^{18}$; and multiple medical contacts in the time after injury. ${ }^{14}$

In an effort to reduce the likelihood of short and long term morbidity, several trials have been undertaken in recent years to evaluate the efficacy of supervised programmes of physiotherapy in improving outcomes of ankle sprains and accelerating return to activity. ${ }^{719-24} \mathrm{~A}$ recent systematic review of these trials identified considerable potential for bias related to methods of randomisation, concealment, and outcome measurement and concluded that current evidence for early implementation of supervised exercise is limited in terms of breadth and quality. ${ }^{25}$ We carried out a randomised clinical trial to address the findings of systematic reviews, including a 2002 Cochrane review, of persisting controversy as to the role of supervised physiotherapy in the management of acute ankle sprains and their call for sufficiently powered trials designed to limit bias. ${ }^{1125}$

\section{Methods}

We undertook a parallel group randomised controlled trial of adults attending the emergency department with ankle injuries. We evaluated the efficacy of a standardised intervention of supervised physiotherapy plus usual emergency medical care versus usual emergency care alone in augmenting functional recovery from grade 1 or 2 ankle sprains at one, three, and six months. Recovery was assessed with a standardised patient assessed outcome score of ankle function - the foot and ankle outcome score (FAOS), ${ }^{26}$ as well as clinical and biomechanical measures of ankle function. The primary outcome of efficacy was defined, a priori, as “excellent” recovery (a total score $\geq 450 / 500$ ) at three months. We hypothesised that a standardised evidence based programme of supervised physiotherapy 20212327 would improve the near term functional outcomes of ankle sprains. In terms of clinical relevance, high quality evidence regarding the efficacy of physiotherapy would assist acute and primary care physicians in providing recommendations for treatment to patients with ankle sprains. ${ }^{2428}$

\section{Participants}

Prospective participants were patients presenting for acute medical treatment of an ankle sprain to one of two acute care settings affiliated with Queen's University in Kingston, Ontario, Canada. The Kingston General Hospital is a 450 bed tertiary care facility providing full emergency department care and the Hotel Dieu Hospital is an ambulatory care hospital with an urgent care setting. Together, these partner facilities manage 95000-100000 encounters with acute care patients each year, of which $20 \%$ are injury related.

We adopted broad inclusion criteria so that our trial cohort was representative of a general adult population presenting for assessment and treatment of ankle sprains. Eligibility criteria were age $\geq 16$; clinical diagnosis of grade 1 or 2 ankle sprain, which could include clinically unimportant avulsion fractures $(<3 \mathrm{~mm}$ of displacement), ${ }^{29}$ with injury sustained less than 72 hours before presentation for assessment; fluency in spoken English; competent to provide informed consent, based on clinical judgment during interview with the patient; willingness to participate in the assessments in the Queen's University motor performance laboratory as per protocol at baseline and at one, three, and six months after injury; and a total FAOS $<450$ measured at the baseline visit in the motor performance laboratory. The exclusion criteria were injury mechanisms that were inconsistent with a ligamentous sprain (such as direct impact); the attending emergency physician determining the need for immobilisation of the injured ankle or surgery based on clinical findings; presentation with concomitant injuries; other mobility limiting conditions; inability to accommodate the time intensive study protocol; and a declared plan to seek physiotherapy for treatment outside the study protocol.

\section{Participant involvement}

No patients were actively involved in setting the research question or the outcome measures nor were they involved in the analysis, interpretation, and writing of the results. Their involvement in the design and implementation of the study was limited to providing informal feedback during a pilot phase to assess their tolerance and acceptance of data collection methods and procedures. Our findings from the trial will be shared with all participants, who will be provided a lay abstract of our study results and conclusions and access to the full manuscript.

\section{Randomisation and follow-up}

Eligible patients identified in the emergency department who were interested in participating in our trial provided consent to have their contact information shared with the research coordinator to schedule a baseline assessment in the motor performance laboratory at Queen's University. Completion of the baseline assessment in the emergency department was impractical as the FAOS is not scored at the time of injury but rather is an assessment of dysfunction, and patients report limitations related to the injury over the previous week. The target appointment time for baseline assessment in the laboratory was, therefore, one week after injury. All potential participants completed both the baseline assessments of the FAOS and clinical and biomechanical measures before randomisation so that they understood the procedures and time commitment involved. The research coordinator then reviewed entry criteria, confirmed eligibility, and obtained informed consent for the study protocol.

Participants' personal information and FAOS were entered into a secure password protected web based central randomisation tool linked to a REDCap study database. ${ }^{30}$ The tool then disclosed the treatment assignment of the current participant to the research coordinator. Participants were randomised to one of two arms of the study: the usual care arm, in which participants received usual care in the emergency 
department, or the physiotherapy arm, in which participants received usual care plus a regimen of supervised physiotherapy. The randomisation tool used a stratified randomisation list created electronically by the study statistician, who was not involved in recruitment or follow-up. A 1:1 allocation in permuted blocks was used within four strata defined by the baseline FAOS, where the four strata were based on the quartiles obtained from pilot study work. The permuted blocks were of variable random size of at most eight, with the size of the blocks unknown to anyone except the trial statistician. An audit trail was recorded, with concealment guaranteed for all future treatment assignments.

Participants assigned to either arm of the trial were booked for a follow-up assessment of outcome variables in the motor performance laboratory one month after the baseline appointment. Participants randomised to the physiotherapy arm additionally had their contact information provided to the physiotherapy clinic at Queen's University. The clinic arranged the first appointment within two business days of the baseline laboratory visit to document history and clinical findings relevant to the care of the injured ankle. Participants allocated to the physiotherapy arm were asked not to reveal this allocation to our research staff.

The research associate who performed the registration and randomisation also coordinated follow-up of participants. Research staff blind to group allocation collected both follow-up and outcome data.

\section{Description of the trial arms}

Participants assigned to the usual care arm received standard emergency department care, consisting of a medical assessment and a one page written summary of instruction for basic management of the injury at home, including ankle protection, rest, cryotherapy (ice), application of a compression bandage, elevation, use of analgesics as necessary, graduated weight bearing activities, and information about expected recovery. No information regarding physiotherapy was discussed with patients in the usual care arm of the study.

Participants assigned to the physiotherapy arm were provided with usual care (as above) plus a regimen of supervised physiotherapy. This intervention included a first appointment to assess the injury and establish a treatment plan and as many as seven subsequent clinic visits at no financial cost to participants. The physiotherapy intervention followed a standardised plan based on current evidence for the use of functional exercises progressed according to four general stages of recovery of ankle sprains ${ }^{2021232731-33}$ : decreased pain and swelling; increased range of motion, strength, and weight bearing; active range of motion and increased exercise tolerance; and restoration of full activities and dynamic ankle stability. Specific treatment goals and therapeutic interventions were provided for each stage of recovery, with clearly defined criteria for progression to the subsequent stage, or discharge. Figure 1 summarises the intervention.

Treatment sessions were 30 minutes in length and were augmented by standardised home exercise plans of active ankle range of motion exercises, isometric strengthening, and resistance exercise, using therapeutic elastic resistance bands or body weight. A home exercise programme was recommended based on each patient's functional recovery. Icing was used when swelling persisted, but no manual therapies or immobilisation (for instance, taping) or brace devices were used. The treatment plan was implemented by one of three assigned physiotherapists, with a single therapist providing all treatment for an individual participant, whenever possible. At each session, the therapist assessed each individual's stage of recovery and recorded compliance with appointments and home exercise programmes.

\section{Outcome measurements}

The FAOS is a self administered questionnaire specific for feet and ankles developed to assess week-to-week change in symptoms and function after foot and ankle injuries. ${ }^{26}$ The FAOS includes 42 items, assessing five domains of recovery from the ankle and/or foot injury: pain, symptoms, function in activities of daily living, function in sport and recreation, and quality of life. ${ }^{2634}$

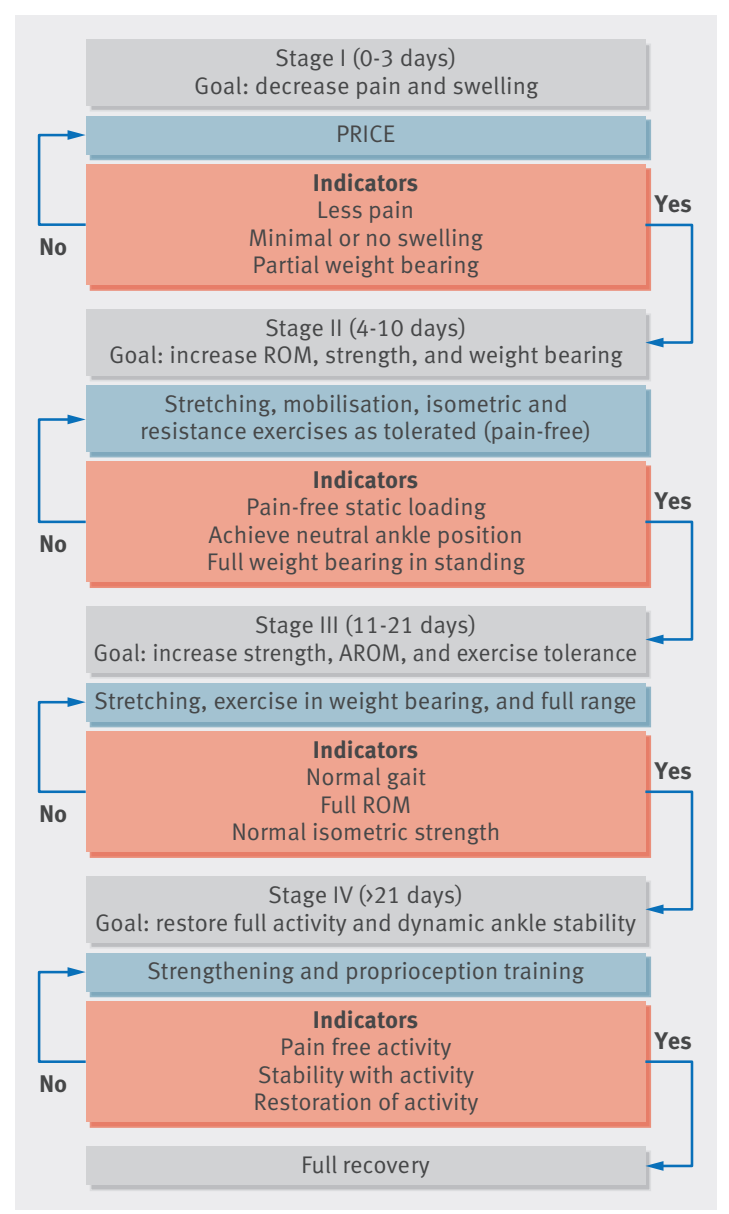

Fig 1 Schematic representation of standardised programme of supervised physiotherapy, showing stages of progression, treatment goals for each stage, and criteria for progression or discharge. PRICE=protection, rest, ice, compression, elevation; $R O M=$ range of motion; AROM=active ROM 
Each scale is scored from 0 to 100 , with a total best possible score of 500 .

The primary outcome of efficacy, stated a priori, was "excellent recovery" from the ankle sprain, defined as a total score $\geq 450 / 500$ at the three month time point of assessment after randomisation. ${ }^{35-37}$

An evidence based FAOS cut off score of excellent recovery has yet to be defined. In our trial, we selected the score of 450/500 based on a previously published study by one of the authors of the FAOS. ${ }^{38}$ Using the ankle function scoring scale, a precursor to the FAOS, Karlsson and Peterson ${ }^{38}$ used a scale score of $90 \%$ as the criterion for excellent recovery, which translates to a FAOS cut off of 450/500. All FAOS outcome data were collected by trained interviewers using a standard interview script and protocol.

Based on the FAOS, we additionally evaluated a series of secondary outcomes, also defined a priori. These included excellent recovery at one and six months (score $\geq 450 / 500$ ) and change from baseline on the total and domain specific FAOS, measured as a continuous variable at one, three, and six months. We included a six month time point of measurement based on previous studies that have shown considerable residual morbidity at six months after injury. ${ }^{39} 40$

We also obtained selected clinical and biomechanical measures for secondary analysis, providing a quantitative assessment of ankle function and recovery from injury. We assessed oedema, quantified using a figure of eight measurement $(\mathrm{mm})^{41}$; manual muscle testing of the ankle dorsiflexors and plantar flexors to provide a grade from 0 (no visible contraction) to 5 (full strength through range of motion); and end range of dorsiflexion, assessed using the lunge test. ${ }^{42}$ We used a Biodex System 3 dynamometer (Biodex Medical Systems, Shirley, NY) to quantify the biomechanical measure ${ }^{43}$ end range (in degrees) of passive and active dorsiflexion and plantar flexion, with the end point of passive range determined either as the point at which resistance reached 5 Newton metre $(\mathrm{Nm})$ or the point at which the patient terminated the movement; and peak dorsiflexion and plantar flexion torque $(\mathrm{Nm})$, defined as the highest torque produced over five dorsiflexion-plantar flexion cycles at a velocity of $30^{\circ} \%$ s. We calculated change in clinical and biomechanical measures of ankle recovery from baseline at one, three, and six months.

Any recurrence of injury of the same ankle was monitored at the one, three, and six month follow-up assessments. Additionally, all participants were asked whether they had accessed any other healthcare provider, including physiotherapy services that were not part of the study.

\section{Statistical analysis}

Sample size

The study protocol targeted enrolment of 256 participants per arm to achieve a power of at least $80 \%$, at a two sided $\alpha$ of 0.05 , to detect an absolute increase in excellent recovery (that is, FAOS $\geq 450$ ) from $60 \%$ to $75 \%$. This sample size estimation includes an inflation factor of 1.63 to allow for intent to treat analysis with a
$15 \%$ loss to follow-up and $15 \%$ treatment cross over. The estimated rate of excellent recovery in the control arm was based on our pilot work. The $15 \%$ difference between arms was based on previous literature and the results of our survey of 22 emergency department clinical physicians, most of whom responded that a $15 \%$ improvement in excellent recovery would be required to justify routine referral to physiotherapy.

\section{Analysis of primary outcome}

Our primary test of efficacy compared the two arms at three months after randomisation using the Mantel-Haenszel test, stratified by baseline total FAOS quarter at a two sided 5\% significance level. We calculated the absolute difference in excellent recovery rates between the two arms, adjusted for baseline quarter, with $95 \%$ confidence intervals.

\section{Analysis of secondary outcomes}

The primary analysis was repeated at one and six months after randomisation. Using continuous FAOS data, we estimated the expected mean changes in total and domain specific score from baseline to one, three, and six months after randomisation by arm, with differences between arms (and 95\% confidence intervals) depicted graphically. These estimates were obtained by restricted maximum likelihood from a repeated measures linear model as implemented in the MIXED procedure of SAS. ${ }^{44}$ This approach used all available assessments including baseline as outcomes, allowing for a flexible (unstructured) covariance structure within individual patients, which is less susceptible to bias from missing data than cross sectional approaches ${ }^{45}$ and is no less effective than using multiple imputation. ${ }^{45-47}$ Predictors in the model included baseline FAOS quarter as a factor, time as a factor, treatment arm as an indicator variable, and the interaction between time and treatment arm to allow the treatment effect to vary over time. We constructed contrasts to estimate the change from baseline within each arm and the difference in the change between arms at one, three, and six months. We evaluated clinical and biomechanical measures from each time point using the same statistical method. The rate of re-injury of the same ankle was evaluated over the six month follow-up, with differences between arms evaluated by $\chi^{2}$ test.

For all outcomes, we performed intent to treat analysis for our primary assessment of efficacy and a supplementary per protocol analysis. The per protocol analysis excluded contamination from participants who were randomised to the physiotherapy arm and did not attend at least one physiotherapy clinic visit in the first three months of the trial, as well as patients randomised to the usual care arm who independently received physiotherapy during the first three months.

We performed the following subgroup analyses: sex, men versus women; age, comparing outcomes for participants aged $\leq 30$ versus $>30$; injury characteristic, non-sports versus sports injury; and grade 1 versus grade 2 sprain. To estimate significance of the subgroup treatment effect modification we used a Wald test of the 


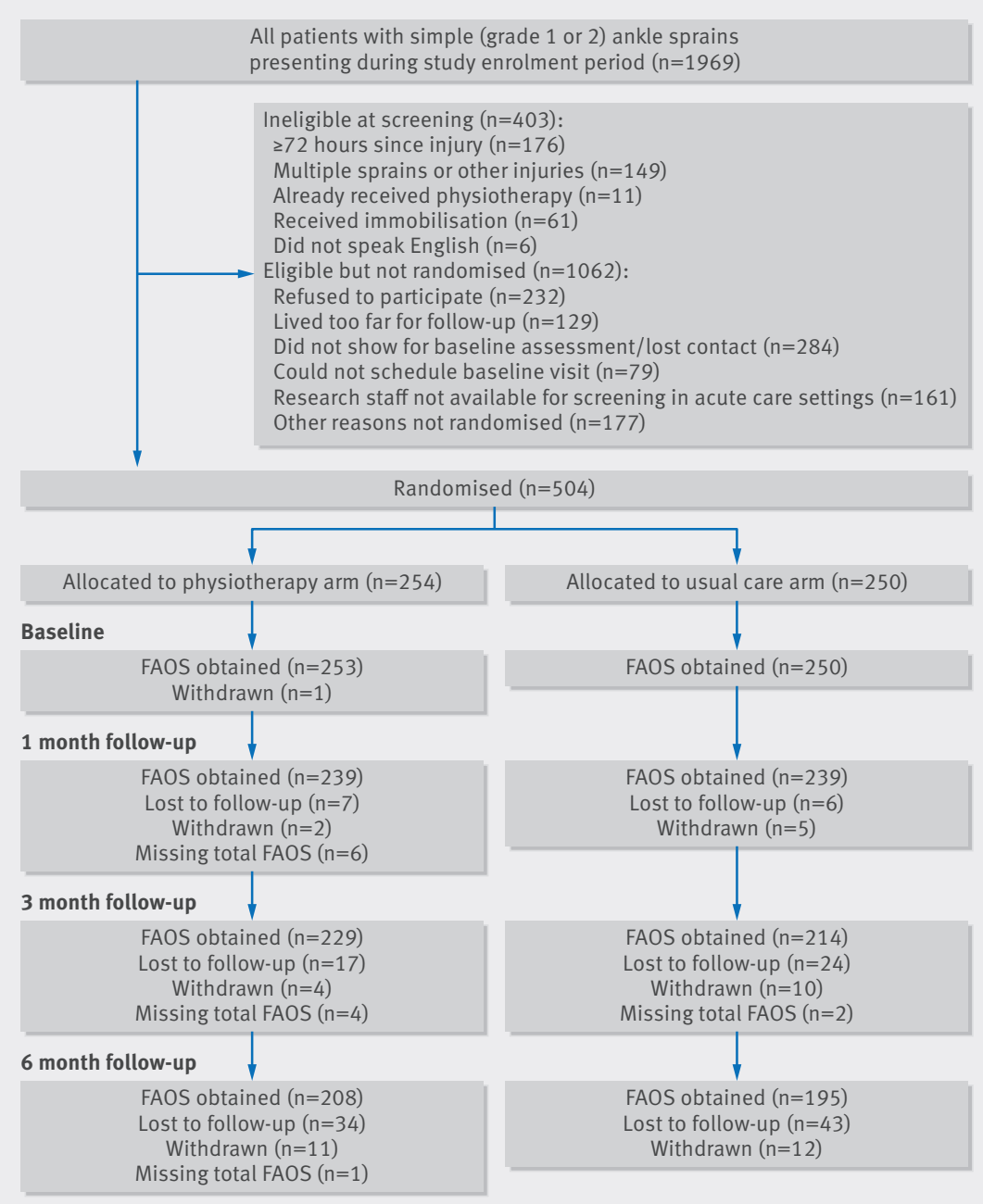

Fig 2 | Patient flow showing loss to follow-up, withdrawal, and missing data by study arm and outcome assessment period. Counts below randomisation are accumulative, with numbers in each box adding up to total number of patients allocated to given arm. Withdrawn=patients who actively refused to participate in further treatment or follow-up; lost to follow-up=patients who could not be contacted for current month assessment; missing FAOS=patients for whom FAOS at specified time point was not obtained

Table 1 | Relevant characteristics of study cohort of patients with acute ankle sprain randomised to usual care with physiotherapy or usual care alone. Figures are numbers (percentage) of patients unless specified otherwise

\begin{tabular}{lll} 
Mean (SD) age (years) & $\begin{array}{l}\text { Physiotherapy } \\
(\mathbf{n = 2 5 3 )}\end{array}$ & $\begin{array}{l}\text { Usual care } \\
(\mathbf{n}=250)\end{array}$ \\
\hline Women & $31.1(13.7)$ & $30.3(13.1)$ \\
\hline Men & $146(58)$ & $134(54)$ \\
\hline Mean (SD) BMI & $107(42)$ & $116(46)$ \\
\hline Grade of sprain: & $28.1(6.3)$ & $28.1(6.8)$ \\
\hline 1 & & \\
\hline 2 & $69(27)$ & $80(32)$ \\
\hline Injured ankle: & $184(73)$ & $170(68)$ \\
\hline$\quad$ Right & & \\
\hline$\quad$ Left & $134(53)$ & $129(52)$ \\
\hline Previous injury to reference ankle & $119(47)$ & $121(48)$ \\
\hline Past surgery on to reference ankle/foot & $152(60)$ & $147(59)$ \\
\hline Plain radiographs ordered at acute care visit & $10(4)$ & $7(3)$ \\
\hline Injured participating in sport & $213(84)$ & $210(84)$ \\
\hline Median (IQR) time (days) between injury and MPL evaluation & $110(43)$ & $105(42)$ \\
\hline Mean (SD) FAOS at baseline (MPL evaluation) & $5(3-6)$ & $5(2-6)$ \\
\hline MPL & $221.6(66.2)$ & $225.4(74.4)$ \\
\hline
\end{tabular}

$\mathrm{MPL}=$ motor performance laboratory; $I Q R=$ interquartile range; $F \mathrm{AOS}=$ foot ankle outcome score. treatment by subgroup interaction term from a logistic regression model with excellent recovery (FAOS $\geq 450$ ) as the outcome variable and baseline FAOS quarter, treatment arm, subgroup indicator, and the treatment by subgroup interaction term as predictors.

To evaluate effects of missing data, we performed a sensitivity analysis, graphically reporting the difference in the three month recovery rate between the two arms of the study over the entire possible range of missing values. We considered the plausibility of missing values being extreme enough to result in a 15\% difference in rate of excellent recovery between the two arms of the study, which we deemed would be large enough to warrant routine referral to physiotherapy. ${ }^{48}$

All analyses were conducted with SAS (version 9.3, SAS Institute, Cary, NC, 2010).

\section{Results}

Between October 2009 and April 2013, we assessed 1969 patients with grade 1 or 2 ankle sprains for eligibility. Of these, 504 patients met our inclusion criteria, provided consent, and were randomised to the physiotherapy $(n=254)$ or the usual care $(n=250)$ arms of our study. One patient in the physiotherapy arm withdrew consent after randomisation and was excluded from the analysis. Figure 2 shows the flow of patients through the study. Table 1 shows relevant patient and injury related characteristics, with no significant differences identified between the two study arms.

Given the actual observed sample size and the rates of contamination and loss to follow-up, the power to detect a 15\% improvement in our primary outcome was $78 \%$ for the intent to treat analyses and $82 \%$ for our per protocol analyses.

Table 2 (intent to treat) and table 3 (per protocol analysis) show comparisons of the study arms for the primary outcome, FAOS $\geq 450$ at three months, and the secondary outcomes of excellent FAOS at one and six months. The per protocol analysis excluded 69 participants in the physiotherapy arm who did not attend at least one protocol physiotherapy session in the first three months, as well as 26 participants in the control arm who received physiotherapy during the first three months. Neither analysis identified strong, consistent, or significant differences in primary or secondary FAOS outcomes by study arm.

Figure 3 (intent to treat analysis) and figure 4 (per protocol analyses) show the change from baseline in total and domain specific continuous FAOS. Differences between groups in the mean change were not significant at any follow-up time point for any FAOS domain. Tables 4 and 5 show the results of the subgroup analysis. Although these indicate a benefit for physiotherapy at three months in the subgroup of patients aged $<30$, with a borderline significant $(\mathrm{P}=0.05)$ interaction between treatment and age at the three month assessment, this trend was not present at other time points and would not remain significant after any reasonable adjustment for multiple testing. There was no significant treatment difference in any other subgroup. Figure 5 characterises the missing data sensitivity anal- 


\begin{tabular}{|c|c|c|c|c|}
\hline \multirow[b]{2}{*}{ Time point } & \multicolumn{2}{|c|}{ No (\%) of patients } & \multirow[b]{2}{*}{ Raw risk difference $(95 \% \mathrm{Cl})$} & \multirow[b]{2}{*}{ Pvalue* } \\
\hline & Physiotherapy & Usual care & & \\
\hline Baseline & $0 / 253(0)$ & $0 / 250(0)$ & - & - \\
\hline 1 month & $26 / 239(11)$ & $34 / 239(14)$ & $-3 \%(-9 \%$ to $3 \%)$ & 0.27 \\
\hline 3 months & $98 / 229(43)$ & $79 / 214(37)$ & $6 \%(-3 \%$ to $15 \%)$ & $0.26 t$ \\
\hline 6 months & $118 / 208(57)$ & $120 / 195(62)$ & $-5 \%(-14 \%$ to $5 \%)$ & $0.26 \dagger$ \\
\hline
\end{tabular}

Table $3 \mid$ Results of per protocol* analysis for primary outcome of excellent recovery (total FAOS $\geq 450$ ) in patients with acute ankle sprain randomised to usual care with physiotherapy or usual care alone

\begin{tabular}{llllll} 
& \multicolumn{2}{l}{ No (\%) of patients } & & \\
\cline { 2 - 3 } Time point & Physiotherapy & Usual care & & Raw risk difference $(95 \% \mathrm{Cl})$ & Pvaluet \\
\hline Baseline & $0 / 184(0)$ & $0 / 224(0)$ & - & - \\
\hline 1 month & $23 / 180(13)$ & $33 / 213(15)$ & $-3 \%(-10 \%$ to $4 \%)$ & 0.65 \\
\hline 3 months & $75 / 177(42)$ & $75 / 188(40)$ & $2 \%(-8 \%$ to $13 \%)$ & 0.42 \\
\hline 6 months & $92 / 165(56)$ & $113 / 174(65)$ & $-9 \%(-20 \%$ to $1 \%)$ & 0.09 \\
\hline
\end{tabular}

*Excludes participants randomised to physiotherapy arm who did not attend at least one protocol physiotherapy session in first 3 months and participants randomised to control arm who received any physiotherapy during first 3 months.

†P value based on Mantel-Haenszel test stratified by quarter of FAOS at baseline.

\section{Mean (SE) FAOS score}

Physio Control

Month 1

Symptoms $20(1) \quad 21(1)$

Pain $\quad 24(1) \quad 22(1)$

Daily living 27 (1) $26(1)$

Sports $\quad 33(1) \quad 32(1)$

Quality of life 23 (1) 23 (1)

Month 3

Overall score $196(5) 188(5)$

Symptoms $32(1) \quad 32(1)$

Pain 35 (1) 34 (1)

Daily living 36 (1) 34 (1)

Sports $\quad 53(2) \quad 50(2)$

Quality of life 40(1) $39(2)$

\section{Month 6}

Overall score 214 (5) $222(5)$

Symptoms $36(1) \quad 38(1)$

Pain $\quad 38(1) \quad 40(1)$

Daily living $37(1) \quad 37(1)$

Sports $\quad 58(2) \quad 59(2)$

Quality of life 45 (2) 47 (2)
Overall score 127 (5) 124 (5)

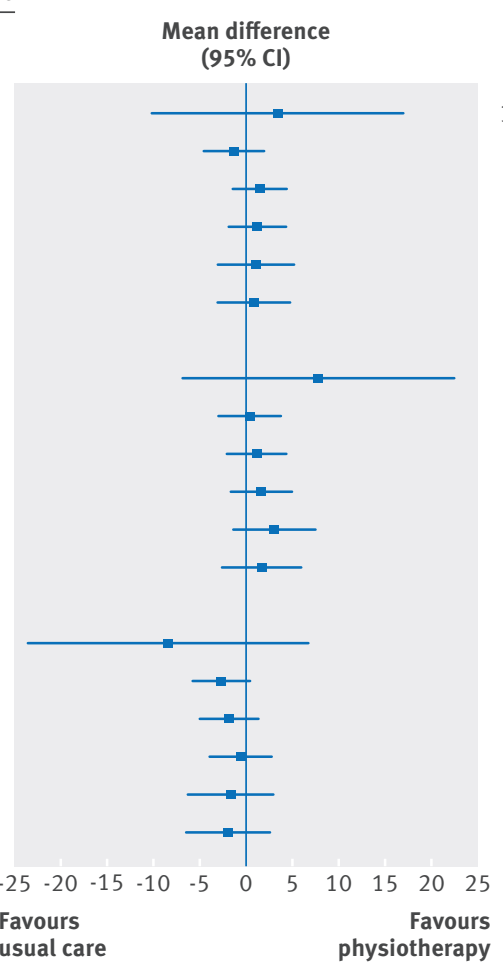

\section{Mean difference} ( $95 \% \mathrm{Cl})$

$3.37(-10.14$ to 16.87$)$ -1.33 (-4.47 to 1.81$)$ $1.43(-1.41$ to 4.26$)$ 1.18 (-1.80 to 4.17$)$ $0.98(-3.07$ to 5.03$)$ $0.81(-2.95$ to 4.58$)$

7.73 (-6.82 to 22.27$)$ 0.37 (-2.84 to 3.57) 1.05 (-1.99 to 4.08$)$ $1.67(-1.47$ to 4.81$)$ 3.05 (-1.30 to 7.39$)$ $1.65(-2.51$ to 5.80$)$

-8.45 ( -23.48 to 6.57$)$ -2.68 ( -5.66 to 0.31$)$ -1.85 (-4.88 to 1.18$)$ $-0.60(-3.83$ to 2.62$)$ -1.66 ( -6.13 to 2.80$)$ $-1.96(-6.34$ to 2.42$)$ excellent recovery and none of the 36 in the usual care arm achieving excellent recovery; or all 25 participants with a missing intervention in the physiotherapy arm and no more than five in the control arm achieving an excellent recovery. Based on the low rate of excellent recovery identified among the $88 \%$ of randomised participants with three month follow-up (tables 2 and 3), these conditions are exceedingly unlikely to be achieved.

Figure 6 (intent to treat analysis) and figure 7 (per protocol analyses) show the assessment of change from baseline in clinical and biomechanical measures at the three time points. In the intent to treat analysis, there were no significant differences among the 10 variables measured. In the per protocol analysis, measures of peak plantar flexor torque $(\mathrm{Nm})$ and plantar flexor power (watts) favoured the physiotherapy arm, with no other clinically important or significant differences identified by treatment arm.

In the six month follow-up period, 19/254 (7.5\%) participants in the physiotherapy arm and 21/250 (8.4\%) in the usual care arm reported a re-injury of the same ankle, with no significant difference between the two trial arms $(\mathrm{P}=0.71)$.

\section{Discussion}

\section{Principal findings}

With the participation of 503 patients, our trial is the largest randomised controlled trial to have evaluated the therapeutic benefits of supervised physiotherapy in the treatment of acute ankle sprains. Based on our outcomes, we conclude that among patients aged 16-79 presenting to an emergency department with a simple grade 1 or 2 ankle sprain within 72 hours of their injury, the addition of early supervised physiotherapy to usual care does not lead to clinically important improvements in functional recovery up to six months after injury, compared with usual care alone. The absence of a therapeutic benefit of supervised exercise was confirmed for our primary outcome of excellent functional recovery as assessed by the FAOS at three months, as well as for the FAOS (using both excellent recovery and continuous scores) and objective clinical and biomechanical measures at one, three, and six months after injury. Our finding is clinically important as the recommendation of physiotherapy for the management of acute ankle sprains comes with substantial economic costs.

\section{Strengths of the trial}

Our trial conformed to the CONSORT guidelines. ${ }^{49}$ Our main findings are unlikely to be explained by imbalances in confounding factors, both known and unknown, between the trial arms. Baseline group comparisons essentially showed equivalence on such factors, suggesting that the approach to randomisation and its administration were effective. Although participants were volunteers, the study population was large and heterogeneous, and, because of the use and effectiveness of randomisation, it is unlikely that any forces of selection resulted in bias in the observed effect esti- (total FAOS 2450 ). To reach a clinically important difference of $15 \%$ favouring the physiotherapy arm, we would have needed either of the following two conditions to be satisfied: at least 20/25 participants with a missing intervention in the physiotherapy arm to have
Fig 3 Intent to treat analysis of change in FAOS from baseline. Estimates are based on restricted maximum likelihood using all available FAOS scores from all patients 


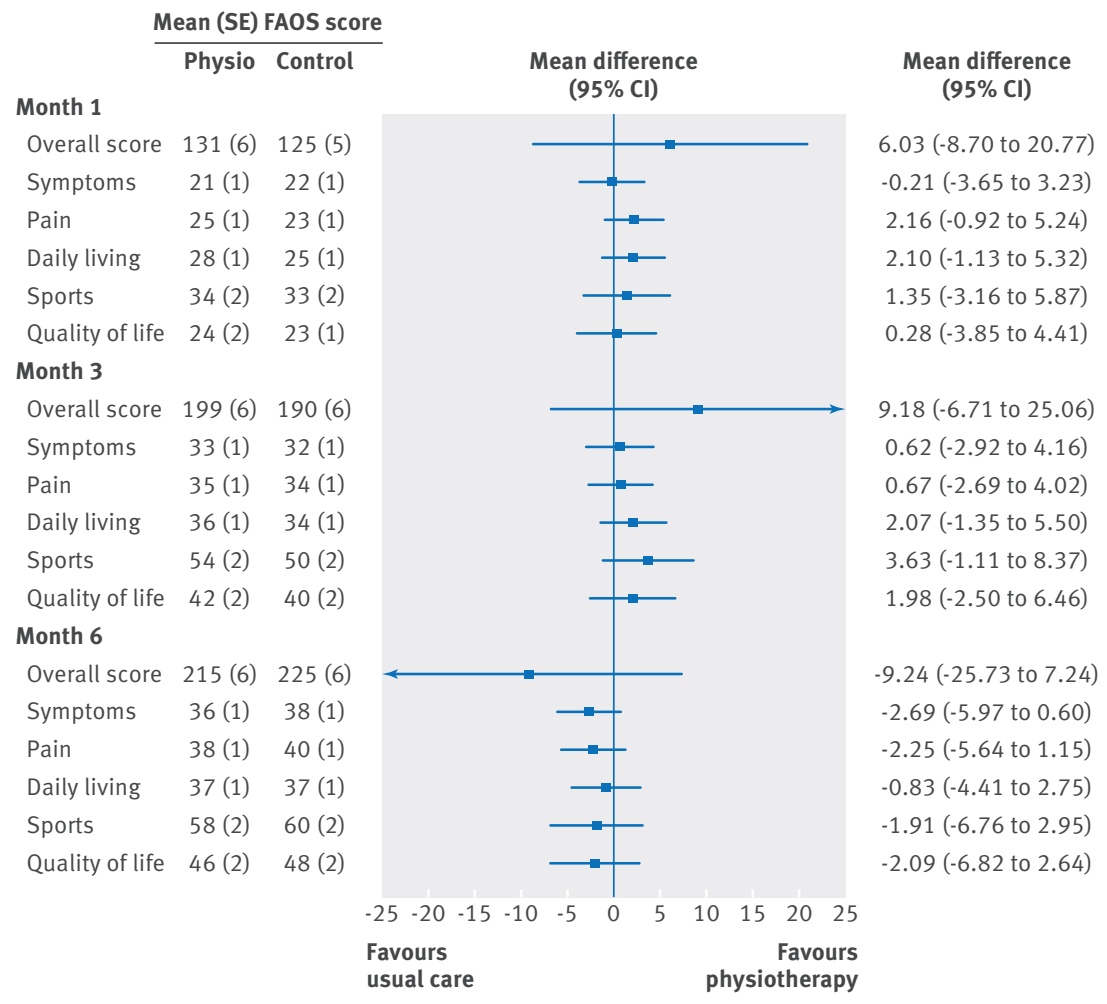

Fig 4 | Per protocol analyses of change in FAOS from baseline. Estimates are based on restricted maximum likelihood using all available FAOS scores from all patients

Table 4 | Subgroup analyses by sex and age for intent to treat analysis of primary outcome of excellent recovery (total FAOS $\geq 450$ ) in patients with acute ankle sprain randomised to usual care with physiotherapy or usual care alone

\begin{tabular}{|c|c|c|c|c|c|}
\hline \multirow[b]{2}{*}{ Subgroup } & \multicolumn{2}{|c|}{ No (\%) of patients } & \multirow{2}{*}{$\begin{array}{l}\text { Raw risk difference* } \\
(95 \% \mathrm{Cl})\end{array}$} & \multirow{2}{*}{$\begin{array}{l}\text { Adjusted risk } \\
\text { difference* } \\
(95 \% \mathrm{Cl}) \dagger\end{array}$} & \multirow[b]{2}{*}{ Pvalue } \\
\hline & Physiotherapy & Usual care & & & \\
\hline \multicolumn{6}{|l|}{ Men } \\
\hline Baseline & $0 / 107(0)$ & $0 / 116(0)$ & - & - & - \\
\hline 1 month & 12/101 (12) & $24 / 112(21)$ & $-10 \%(-19 \%$ to $0 \%)$ & $-7 \%(-20 \%$ to $6 \%)$ & 0.09 \\
\hline 3 months & 46/93 (49) & 43/94 (46) & $4 \%(-11 \%$ to $18 \%)$ & $4 \%(-10 \%$ to $17 \%)$ & 0.60 \\
\hline 6 months & $56 / 88(64)$ & $54 / 86(63)$ & $1 \%(-13 \%$ to $15 \%)$ & $2 \%(-12 \%$ to $15 \%)$ & 0.81 \\
\hline \multicolumn{6}{|l|}{ Women } \\
\hline Baseline & $0 / 146(0)$ & $0 / 134(0)$ & - & - & - \\
\hline 1 month & 14/138 (10) & 10/127 (8) & $2 \%(-5 \%$ to $9 \%)$ & $2 \%(-5 \%$ to $8 \%)$ & 0.59 \\
\hline 3 months & $52 / 136(38)$ & $36 / 120(30)$ & $8 \%(-3 \%$ to $20 \%)$ & $7 \%(-3 \%$ to $18 \%)$ & 0.25 \\
\hline 6 months & $62 / 120(52)$ & $66 / 109$ (61) & $-9 \%(-22 \%$ to $4 \%)$ & $-10 \%(-23 \%$ to $2 \%)$ & 0.12 \\
\hline \multicolumn{6}{|l|}{ Age $\leq 30$} \\
\hline Baseline & $0 / 145(0)$ & $0 / 150(0)$ & - & - & - \\
\hline 1 month & 18/139 (13) & $22 / 143(15)$ & $-2 \%(-11 \%$ to $6 \%)$ & $-1 \%(-8 \%$ to $6 \%)$ & 0.47 \\
\hline 3 months & $68 / 132(52)$ & 47/122(39) & $13 \%$ (1\% to $5 \%)$ & $14 \%$ (3\% to $25 \%)$ & $0.03^{\star \star}$ \\
\hline 6 months & 75/118 (64) & $74 / 106(70)$ & $-6 \%(-19 \%$ to $6 \%)$ & $-6 \%(-17 \%$ to $6 \%)$ & 0.37 \\
\hline \multicolumn{6}{|l|}{ Age $>30$} \\
\hline Baseline & $0 / 108(0)$ & $0 / 100(0)$ & - & - & - \\
\hline 1 month & $8 / 144$ (8) & 12/96 (13) & $-5 \%(-13 \%$ to $4 \%)$ & $-3 \%(-14 \%$ to $8 \%)$ & 0.43 \\
\hline 3 months & $30 / 97(31)$ & $32 / 92(35)$ & $-4 \%(-17 \%$ to $10 \%)$ & $-4 \%(-16 \%$ to $9 \%)$ & 0.52 \\
\hline 6 months & 43/90 (48) & $46 / 89(52)$ & $-4 \%(-19 \%$ to $11 \%)$ & $-6 \%(-19 \%$ to $6 \%)$ & 0.52 \\
\hline
\end{tabular}

*Positive risk difference favours physiotherapy arm.

tAdjusted risk difference uses generalised linear model with binomial outcome and identity link to control for quarter of FAOS at baseline.

¥P value based on Mantel-Haenszel test stratified by quarter of FAOS at baseline.

§P value for subgroup by treatment interaction 0.14 at 1 month, 0.71 at 3 months, and 0.21 at 6 months.

IP value for subgroup by treatment interaction 0.73 at 1 month, 0.05 at 3 months, and 0.93 at 6 months.

${ }^{\star} *$ Discrete treatment effect of $\mathrm{P}<0.05$ : these effects were not consistent across time points of measurement within the subgroups of age $\leq 30$ and $>30$, and would not remain significant after any reasonable adjustment of

multiple comparisons. mates. A participation rate of $88 \%$ at the three month primary endpoint and $80 \%$ at six months was maintained. Subgroup analyses suggested a benefit of physiotherapy at three months in younger patients (aged $<30$ ). The direction of effect was not consistent, however, and favoured the usual care group at one and six months. There were no significant differences between study arms in subgroup analyses by sex, cause of injury (such as sports related or not), and grade of injury (grade 1 or 2) at one, three, and six months after injury.

We also conducted a sensitivity analysis, as recommended by Thabane and colleagues, ${ }^{50}$ to verify the robustness of our findings. Consideration of missing data from loss to follow-up, by re-estimating the treatment effect of the primary outcome over the entire possible range of missing values, did not change our findings. We identified contamination, from non-compliance with the trial protocol in terms of not receiving assigned $(27.3 \%)$ physiotherapy or by independently seeking physiotherapy when not assigned (10.4\%) and conducted separate intent to treat and per protocol analyses. Both analyses failed to show clinically important effects associated with the physiotherapy intervention, and the observed trends reversed from three months to six months.

Another potential source of bias is the effect of "social desirability," where patients assigned to an active intervention are more prone to self report favourable outcomes during clinical interviews. ${ }^{51}$ Such bias would be expected to favour the physiotherapy intervention. There were, however, no clinically 


\begin{tabular}{|c|c|c|c|c|c|}
\hline \multirow[b]{2}{*}{ Follow-up } & \multicolumn{2}{|c|}{ No (\%) of patients } & \multirow{2}{*}{$\begin{array}{l}\text { Raw risk difference* } \\
(95 \% \mathrm{CI})\end{array}$} & \multirow{2}{*}{$\begin{array}{l}\text { Adjusted risk } \\
\text { difference* }(95 \% \mathrm{CI}) \dagger\end{array}$} & \multirow[b]{2}{*}{ P value $\neq$} \\
\hline & Physiotherapy & Usual care & & & \\
\hline \multicolumn{6}{|c|}{ Non-sports injury } \\
\hline Baseline & $0 / 143(0)$ & $0 / 145(0)$ & - & - & - \\
\hline 1 month & $13 / 135(10)$ & 18/137 (13) & $-4 \%(-11 \%$ to $4 \%)$ & $-2 \%(-9 \%$ to $4 \%)$ & 0.32 \\
\hline 3 months & $56 / 131(43)$ & $48 / 124(39)$ & $4 \%(-8 \%$ to $16 \%)$ & $3 \%(-8 \%$ to $14 \%)$ & 0.66 \\
\hline 6 months & $63 / 117(54)$ & $70 / 112(63)$ & $-9 \%(-21 \%$ to $4 \%)$ & $-9 \%(-21 \%$ to $3 \%)$ & 0.13 \\
\hline \multicolumn{6}{|c|}{ Sports injury } \\
\hline Baseline & $0 / 110(0)$ & $0 / 105(0)$ & - & - & - \\
\hline 1 month & 13/104 (13) & 16/102 (16) & $-3 \%(-13 \%$ to $6 \%)$ & $-0 \%(-8 \%$ to $7 \%)$ & 0.61 \\
\hline 3 months & 42/98 (43) & $31 / 90(34)$ & $8 \%(-5 \%$ to $22 \%)$ & $9 \%(-3 \%$ to $22 \%)$ & 0.22 \\
\hline 6 months & $55 / 91(60)$ & $50 / 83(60)$ & $0 \%(-14 \%$ to $15 \%)$ & $0 \%(-14 \%$ to $14 \%)$ & 0.93 \\
\hline \multicolumn{6}{|l|}{ Grade 1} \\
\hline Baseline & $0 / 69(0)$ & $0 / 80(0)$ & - & - & - \\
\hline 1 month & $10 / 66(15)$ & $15 / 75(20)$ & $-5 \%(-17 \%$ to $8 \%)$ & $2 \%(-13 \%$ to $16 \%)$ & 0.37 \\
\hline 3 months & $38 / 67(57)$ & $35 / 68(51)$ & $5 \%(-12 \%$ to $22 \%)$ & $4 \%(-12 \%$ to $21 \%)$ & 0.60 \\
\hline 6 months & $43 / 60(72)$ & $46 / 64(72)$ & $-0 \%(-16 \%$ to $16 \%)$ & $-2 \%(-18 \%$ to $13 \%)$ & 0.89 \\
\hline \multicolumn{6}{|l|}{ Grade 2} \\
\hline Baseline & $0 / 184(0)$ & $0 / 170(0)$ & - & - & - \\
\hline 1 month & $16 / 173(9)$ & $19 / 164(12)$ & $-2 \%(-9 \%$ to $4 \%)$ & $-2 \%(-7 \%$ to $3 \%)$ & 0.42 \\
\hline 3 months & $60 / 162(37)$ & $44 / 146(30)$ & $7 \%(-4 \%$ to $17 \%)$ & $6 \%(-3 \%$ to $16 \%)$ & 0.26 \\
\hline 6 months & $75 / 148(51)$ & $74 / 131(56)$ & $-6 \%(-18 \%$ to $6 \%)$ & $-6 \%(-18 \%$ to $5 \%)$ & 0.26 \\
\hline \multicolumn{6}{|c|}{$\begin{array}{l}\text { *Positive risk difference favours physiotherapy arm. } \\
\text { †Adjusted risk difference uses generalised linear model with binomial outcome and identity link to control for quarter of FAOS at baseline. } \\
\text { †P value based on Mantel-Haenszel test, stratified by quarter of FAOS at baseline. } \\
\text { §P value for subgroup by treatment interaction } 0.77 \text { at } 1 \text { month, } 0.50 \text { at } 3 \text { months, and } 0.27 \text { at } 6 \text { months. } \\
\text { IP value for subgroup by treatment interaction } 0.97 \text { at } 1 \text { month, } 0.85 \text { at } 3 \text { months, and } 0.59 \text { at } 6 \text { months. }\end{array}$} \\
\hline
\end{tabular}

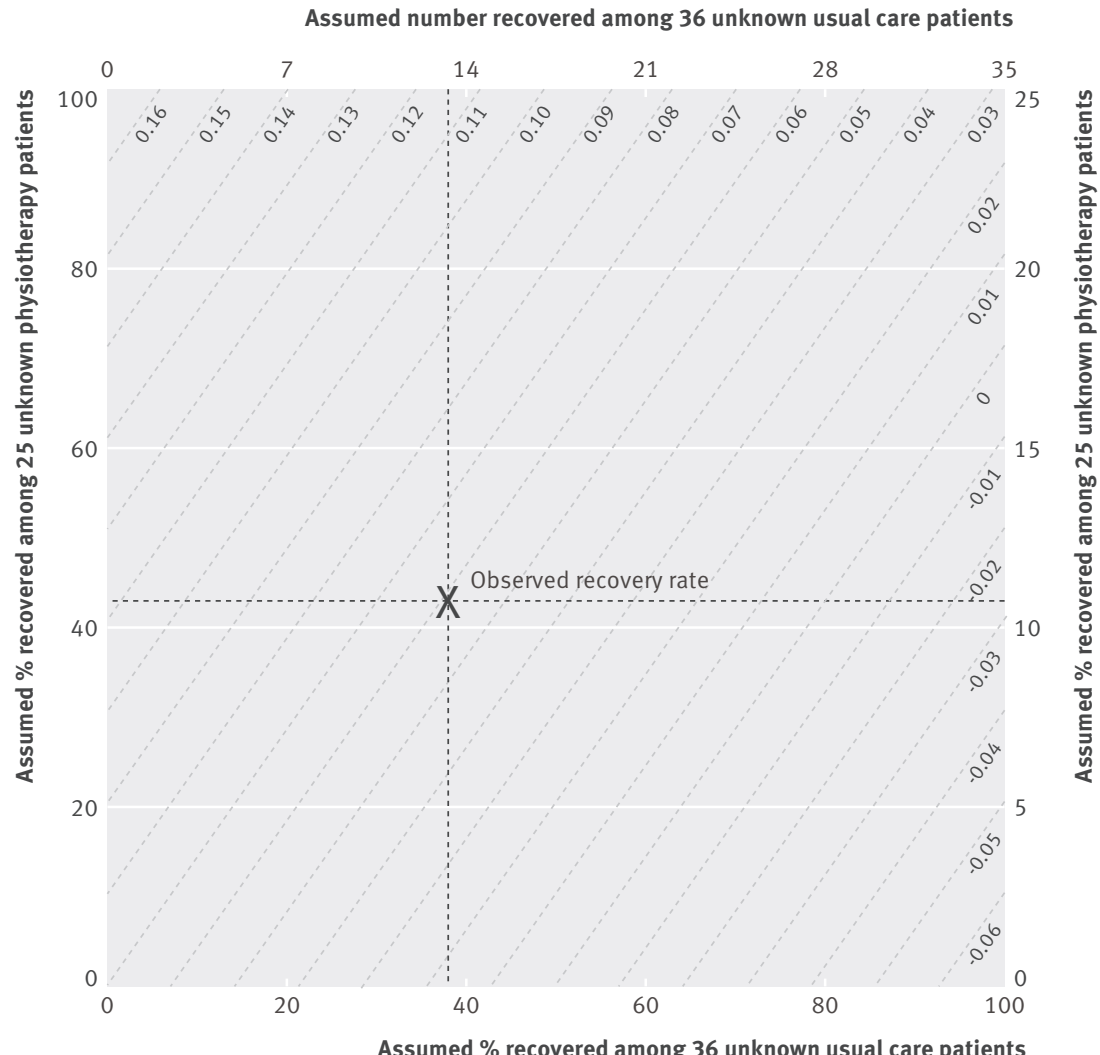

Fig 5 | Sensitivity analysis for missing FAOS data to assess "excellent" recovery at three months, plotting predicted proportion of recovery among 25 unknown participants in physiotherapy arm to predicted proportion among 36 unknown participants in control arm. Main axes (bottom and left) show percentages and secondary axes (top and right) show counts important differences or consistent trends in outcomes over time for both the self reported FAOS and the clinical and biomechanical measures of ankle function. Blinding was implemented when possible among the attending therapists, laboratory personnel, and interviewers.

The FAOS is one outcome measurement available to determine patient assessed foot and ankle function. ${ }^{24}$ We do recognise that although the FAOS has been refined and validated in various contexts, ${ }^{315253}$ the nature and extent of non-differential misclassification of the primary study outcome remains unknown. Therefore, any misclassification of outcomes in our trial is most likely to be non-differential in nature. Also, our criterion of a score $\geq 450$ denoting excellent recovery was based on studies that used similar self report questionnaires for lower extremities-namely, the lower extremity function score (LEFS) ${ }^{54}$ and the knee injury and osteoarthritis outcome score (KOOS). 48 The evaluation of the FAOS as a continuous variable, as we did in our secondary analyses, allows for a more statistically efficient analysis of this outcome. The use of a cut off to dichotomise recovery as "excellent" or "not excellent," however, provides a more clinically interpretable outcome. ${ }^{55}$ It is likely that patient assessed measures of excellent recovery will be influenced by the characteristics of the clinical population under study, ${ }^{25}$ and, therefore, a range of cut offs might be appropriate. Identification of those characteristics that modify patients' assessment of recovery requires further research, though the stringent criterion adopted in our study provides confidence that patients 


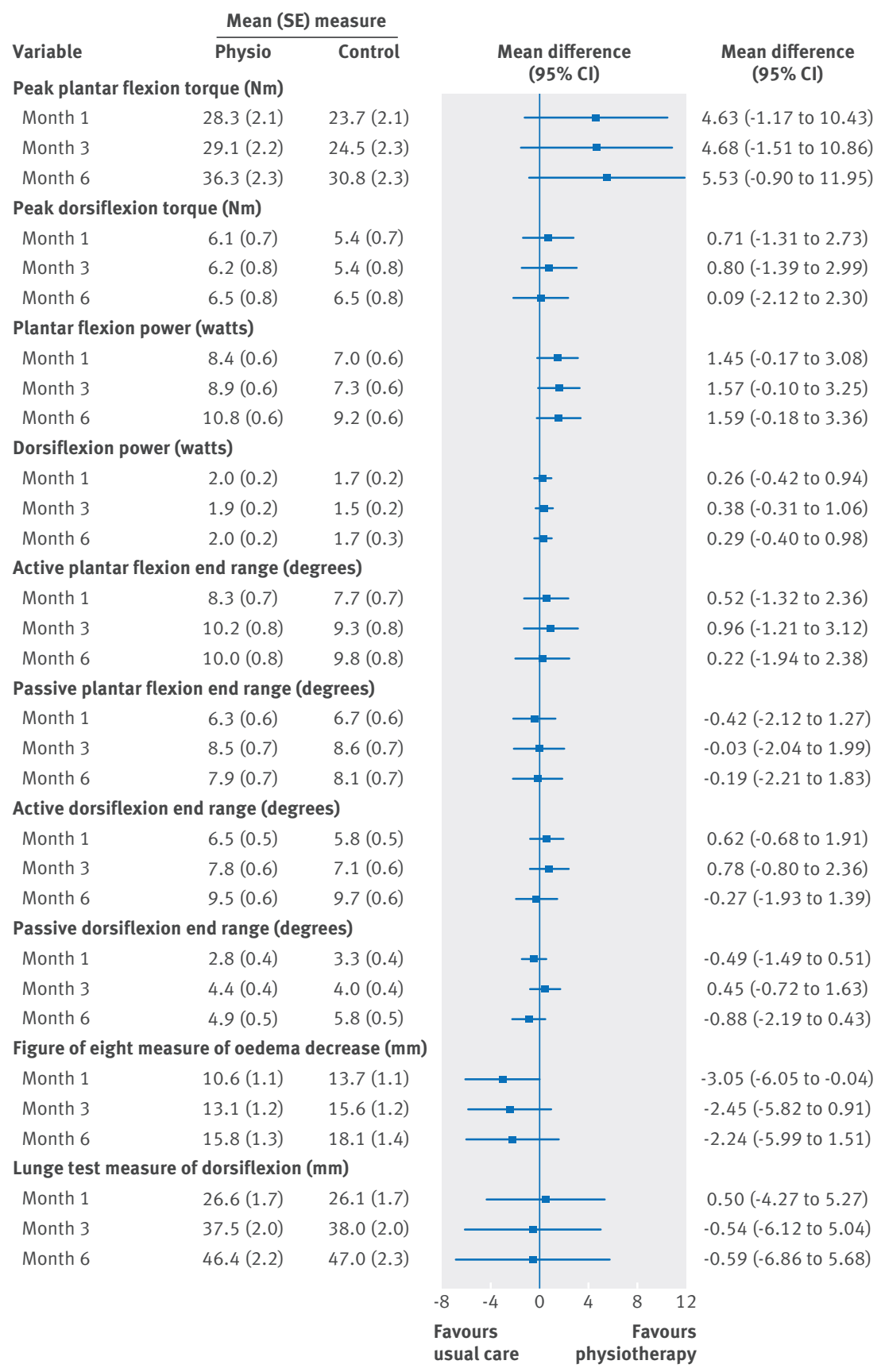

Fig 6 | Intent to treat analysis of change in clinical and biomechanical measures from baseline. All estimates reported as increase from baseline, with exception of figure of eight measure of oedema, which is reported as decrease from baseline, so that greater increase is consistently favourable. Estimates based on restricted maximum likelihood using all available measures from all randomised participants

classified as recovered were appropriately categorised as recovered. ${ }^{135657}$

\section{Comparison with other studies}

Our results contradict those of Hultman and colleagues, ${ }^{24}$ who conducted a randomised controlled trial to evaluate the effectiveness of providing four physiotherapy visits in addition to usual emergency department care on recovery of ankle function measured at six weeks and at three months after injury. The physiotherapy visits were held within 14 days of attendance. As in our study, the physiotherapy regimen was standardised and based on functional exercises, using the FAOS as the primary outcome. Hultman and colleagues reported a therapeutic benefit of physiotherapy in addition to information provided in the department. Their trial was relatively small, with 33 patients in the intervention group and 32 in the control group. Their protocol instructed patients to exercise to a pain level of 5 out of 10 on a visual analogue scale, according to Thomée's model $^{57}$ of pain control, whereas our patients were instructed to exercise as tolerated. The role of training to 


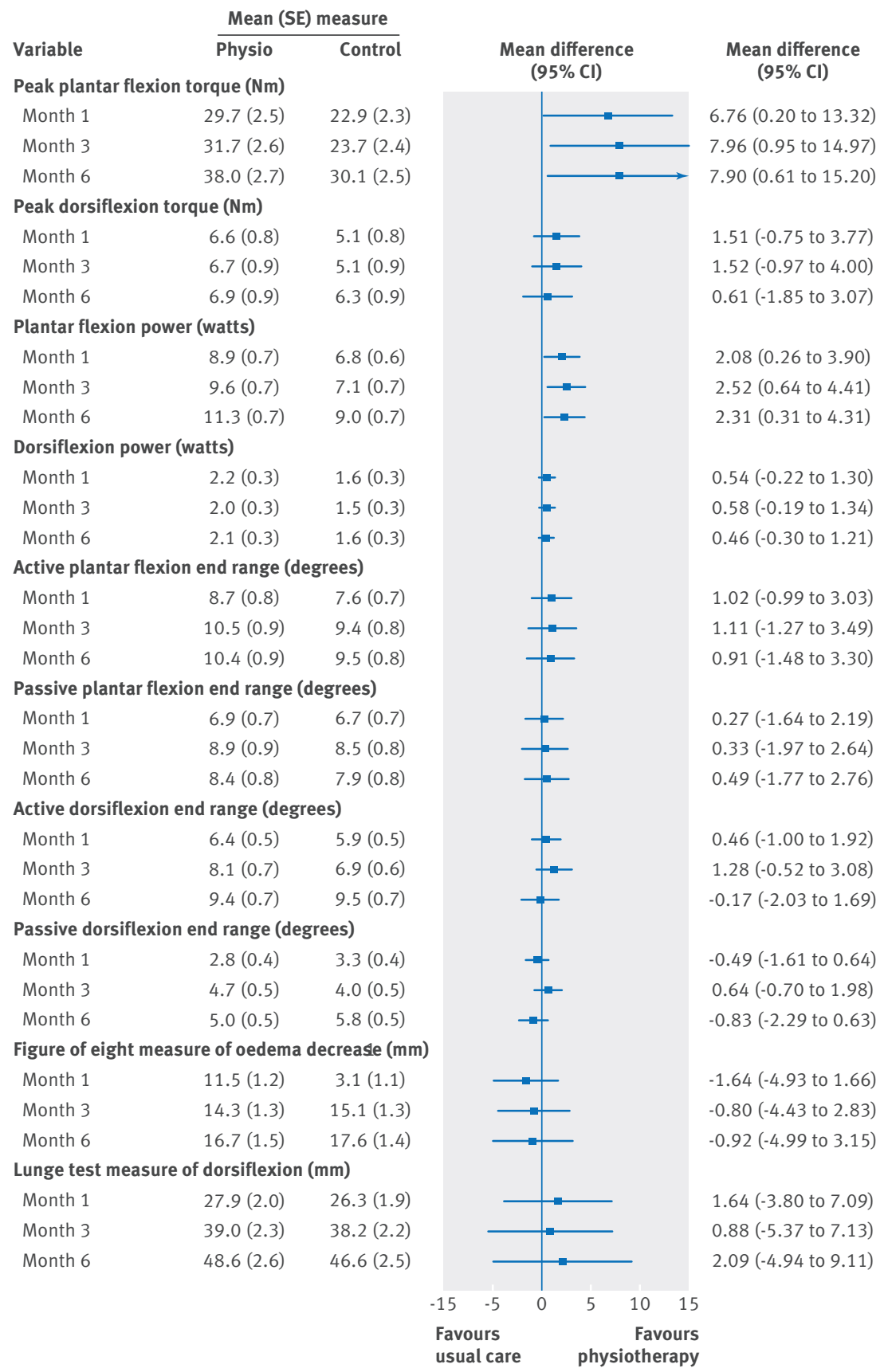

Fig 7 | Per protocol analyses of change in clinical and biomechanical measures from baseline. All estimates reported as increase from baseline, with exception of figure of eight measure of oedema, which is reported as decrease from baseline, so that greater increase is consistently favourable. Estimates based on restricted maximum likelihood using all available measures from all randomised participants

a higher level of pain, and the reinforcement of this during physiotherapy, could warrant further evaluation.

\section{Limitations of the trial}

The limitations of our trial need to be acknowledged in the interpretation and application of our findings to practice. Our extensive protocol might have introduced bias in the characteristics of participants in our study group. While we were successful in enrolling 504 patients, this represents a recruitment rate of one in three patients who met our clinical criteria of eligibility.
We suspect that our time intensive appointments for outcome assessment might have contributed to the refusal of 232 eligible patients to participate. Another 129 eligible patients declined to participate because of travel distance, a consequence of our urgent care centres serving a large geographical region. As such, our sample might not be generalisable to all those who experience grade 1 and 2 ankle injuries. Our high retention at the three month primary end point $(88 \%)$ and at six months (80\%) supports a high level of commitment among our study participants. 


\section{Conclusions and implications for practice}

Our trial provides level I evidence that supervised physiotherapy, as described, does not provide clinically important benefit in the management of simple ankle sprains in a general population of patients seeking care in an acute care setting. Our comparator of usual care was the provision of written recommendations for initial rest, cryotherapy, compression, and limb elevation, with graduated mobility being encouraged as tolerated. These recommendations stem from basic principles of physiotherapy, which, to our knowledge, are commonly provided in emergency departments. This format of usual care is associated with minimal cost and is unlikely to ever be assessed against no care. We do note that $43 \%$ of participants in the physiotherapy arm and 38\% in the control arm had not reached excellent recovery by six months; this being lower than anticipated. This suggests that while there was not a clinically important effect with the standardised physiotherapy regimen provided to our participants, there is potential for the investigation of alternative interventions that would reduce morbidity in patients with these injuries.

We thank D Emerton, who coordinated data collection, X Sun, who assisted with data analysis, S Dostaler, who assisted with trial development and registration, and I Bielska for maintaining literature reviews. We also thank all participants for their time and commitment to our study and participants involved in the pilot phase of our trial, who provided feedback on our methods and procedures.

Contributors: RJB and AGD were responsible for data integrity and analyses for the study. All authors contributed to the study concept and design. RJB and BB were responsible for data acquisition. AGD, $\mathrm{RJB}, \mathrm{BB}$, and WP were responsible for the statistical analysis and interpretation. RJB, AGD, LP, BB, and WP drafted the manuscript. All authors contributed to the critical revision of the manuscript for intellectual content. Study supervision was provided by RJB and BB. $\mathrm{RJB}$ is guarantor.

Funding: The study was funded by the Canadian Institutes of Health Research (MCT-94833). The sponsors had no role in the design, conduct, and analysis or involvement in the preparation or submission of the manuscript for publication

Competing interests: All authors have completed the ICMJE uniform disclosure form at www.icmje.org/coi disclosure.pdf and declare: no support from any organisation for the submitted work; no financial relationships with any organisations that might have an interest in the submitted work in the previous three years; no other relationships or activities that could appear to have influenced the submitted work. Ethical approval: This study was approved by Queen's University Health Sciences Research Ethics Board (file No 6004187) and informed consent was given by all patients.

Data sharing: The investigators will share data (with associated coding library) used in developing the results presented in this manuscript on request (from AGD, daya@kgh.kari.net). Anonymised record level data would be made available on request to investigators who have submitted a publicly available proposal for analysis and who have received ethical clearance from their host institution.

Transparency: The lead author affirms that this manuscript is an honest, accurate, and transparent account of the study being reported; that no important aspects of the study have been omitted; and that any discrepancies from the study as planned (and, if relevant, registered) have been explained.

This is an Open Access article distributed in accordance with the Creative Commons Attribution Non Commercial (CC BY-NC 3.0) license, which permits others to distribute, remix, adapt, build upon this work non-commercially, and license their derivative works on different terms, provided the original work is properly cited and the use is non-commercial. See: http://creativecommons.org/licenses/ by-nc/3.0/.

1 Waterman BR, Owens BD, Davey S, Zacchilli MA, Belmont PJ Jr. The epidemiology of ankle sprains in the United States. J Bone Joint Surg Am 2010;92:2279-84. doi:10.2106/JBJS.I.01537.
2 Bridgman SA, Clement D, Downing A, Walley G, Phair I, Maffulli N. Population based epidemiology of ankle sprains attending accident and emergency units in the West Midlands of England, and a survey of UK practice for severe ankle sprains. Emerg Med / 2003;20:508-10. doi:10.1136/emj.20.6.508.

3 Leininger RE, Knox CL, Comstock RD. Epidemiology of 1.6 million pediatric soccer-related injuries presenting to US emergency departments from 1990 to 2003. Am J Sports Med 2007;35:288-93. doi:10.1177/0363546506294060

4 Tadros AMA, Eid HO, Abu-Zidan FM. Epidemiology of foot injury in a high-income developing country. Injury 2010;41:137-40. doi:10.1016/j.injury.2009.05.031.

5 Lambers K, Ootes D, Ring D. Incidence of patients with lower extremity injuries presenting to US emergency departments by anatomic region, disease category, and age. Clin Orthop Relat Res 2012;470:284-90. doi:10.1007/s11999-011-1982-z.

6 Backx FJ, Erich WB, Kemper AB, Verbeek AL. Sports injuries in school-aged children. An epidemiologic study. Am / Sports Med 1989;17:234-40. doi:10.1177/036354658901700214

7 Wolfe MW, Uhl TL, Mattacola CG, McCluskey LC. Management of ankle sprains. Am Fam Physician 2001;63:93-104.

8 Milgrom C, Shlamkovitch N, Finestone A, et al. Risk factors for lateral ankle sprain: a prospective study among military recruits. Foot Ankle 1991:12:26-30. doi:10.1177/107110079101200105.

9 Karlsson J, Eriksson BI, Swärd L. Early functional treatment for acute ligament injuries of the ankle joint. Scand J Med Sci Sports 1996:6:341-5. doi:10.1111/j.1600-0838.1996.tb00104.x.

10 Kerkhoffs GMMJ, Struijs PAA, Marti RK, Assendelft WJJ, Blankevoort L, van Dijk CN. Different functional treatment strategies for acute lateral ankle ligament injuries in adults. Cochrane Database Syst Rev 2002;(3):CD002938. doi:10.1002/14651858.CD002938.

11 Kerkhoffs GMMJ, Rowe BH, Assendelft WJJ, Kelly K, Struijs PAA, van Dijk CN. Immobilisation and functional treatment for acute lateral ankle ligament injuries in adults. Cochrane Database Syst Rev 2002;(3):CD003762. doi:10.1002/14651858.CD003762.

12 Kerkhoffs GMMJ, Handoll HHG, de Bie R, Rowe BH, Struijs PAA. Surgical versus conservative treatment for acute injuries of the lateral ligament complex of the ankle in adults. Cochrane Database Syst Rev 2007;(2):CD000380. doi:10.1002/14651858. CD000380.pub2.

13 Anandacoomarasamy A, Barnsley L. Long term outcomes of inversion ankle injuries. Br J Sports Med 2005;39:e14, discussion e14. doi:10.1136/bjsm.2004.011676.

14 Birrer RB, Fani-Salek MH, Totten VY, Herman LM, Politi V. Managing ankle injuries in the emergency department. J Emerg Med 1999:17:651-60. doi:10.1016/S0736-4679(99)00060-8.

15 Braun BL. Effects of ankle sprain in a general clinic population 6 to 18 months after medical evaluation. Arch Fam Med 1999;8:143-8. doi:10.1001/archfami.8.2.143.

16 Leanderson J, Wredmark T. Treatment of acute ankle sprain. Comparison of a semi-rigid ankle brace and compression bandage in 73 patients. Acta Orthop Scand 1995;66:529-31. doi:10.3109/17453679509002308.

17 Audenaert A, Prims J, Reniers GLL, Weyns D, Mahieu P, Audenaert E. Evaluation and economic impact analysis of different treatment options for ankle distortions in occupational accidents. J Eval Clin Pract 2010:16:933-9. doi:10.1111/j.1365-2753.2009.01231.x.

18 Cooke MW, Marsh JL, Clark M, et al. CAST trial group. Treatment of severe ankle sprain: a pragmatic randomised controlled trial comparing the clinical effectiveness and cost-effectiveness of three types of mechanical ankle support with tubular bandage. The CAST trial. Health Technol Assess 2009;13:iii, ix-x, 1-121. doi:10.3310/ hta13130.

19 Weinstein ML. An ankle protocol for second-degree ankle sprains. Mil Med 1993;158:771-4.

20 Cooke MW, Lamb SE, Marsh J, Dale J. A survey of current consultant practice of treatment of severe ankle sprains in emergency departments in the United Kingdom. Emerg Med J 2003;20:505-7. doi:10.1136/emj.20.6.505.

21 Safran MR, Zachazewski JE, Benedetti RS, Bartolozzi AR 3rd, , Mandelbaum R. Lateral ankle sprains: a comprehensive review part 2 treatment and rehabilitation with an emphasis on the athlete. Med Sci Sports Exerc 1999;31(Suppl):S438-47. doi:101097/00005768-199907001-00005.

22 Bleakley CM, O'Connor SR, Tully MA, et al. Effect of accelerated rehabilitation on function after ankle sprain: randomised controlled trial. BMJ 2010;340:c1964. doi:10.1136/bmi.c1964.

23 van Rijn RM, van Heest JAC, van der Wees P, Koes BW, Bierma-Zeinstra SMA. Some benefit from physiotherapy intervention in the subgroup of patients with severe ankle sprain as determined by the ankle function score: a randomised trial. Aust J Physiother 2009;55:107-13. doi:10.1016/S0004-9514(09)70040-6.

24 Hultman K, Faltstrom A, Oberg U. The effect of early physiotherapy after an acute ankle sprain. Adv Physiother 2010;12:65-73doi:10.3109/14038190903174262. 
25 van Rijn RM, van Ochten J, Luijsterburg PA, van Middelkoop M, Koes BW, Bierma-Zeinstra SMA. Effectiveness of additional supervised exercises compared with conventional treatment alone in patients with acute lateral ankle sprains: systematic review. $B M$ 2010;341:c5688. doi:10.1136/bmj.c5688.

26 Roos EM. A User's Guide to: Foot and Ankle Outcome Score (FAOS). Foot and Ankle Outcome Score, 2003.

27 Gerber JP, Williams GN, Scoville CR, Arciero RA, Taylor DC. Persistent disability associated with ankle sprains: a prospective examination of an athletic population. Foot Ankle Int 1998:19:653-60. doi:10.1177/107110079801901002.

28 Chorley JN. Ankle sprain discharge instructions from the emergency department. Pediatr Emerg Care 2005;21:498-501. doi:10.1097/01. pec.0000173346.15154.f3.

29 Stiell IG, McDowell I, Nair RC, et al. Use of radiography in acute ankle injuries: physicians' attitudes and practice. CMA/ 1992;147:1671-8.

30 Harris PA, Taylor R, Thielke R, Payne J, Gonzalez N, Conde JG. Research electronic data capture (REDCap)--a metadata-driven methodology and workflow process for providing translational research informatics support. / Biomed Inform 2009:42:377-81. doi:10.1016/j. jbi.2008.08.010.

31 Mattacola CG, Dwyer MK. Rehabilitation of the ankle after acute sprain or chronic instability. J Athl Train 2002:37:413-29.

32 Zöch C, Fialka-Moser V, Quittan M. Rehabilitation of ligamentous ankle injuries: a review of recent studies. Br J Sports Med 2003;37:291-5. doi:10.1136/bjsm.37.4.291

33 Ardèvol I, Bolíbar I, Belda V, Argilaga S. Treatment of complete rupture of the lateral ligaments of the ankle: a randomized clinical trial comparing cast immobilization with functional treatment. Knee Surg Sports Traumatol Arthrosc 2002;10:371-7. doi:10.1007/s00167-002-0308-9.

34 Roos EM, Brandsson S, Karlsson J. Validation of the foot and ankle outcome score for ankle ligament reconstruction. Foot Ankle Int 2001;22:788-94.

35 Haywood KL, Hargreaves J, Lamb SE. Multi-item outcome measures for lateral ligament injury of the ankle: a structured review. J Eval Clin Pract 2004;10:339-52. doi:10.1111/j.1365-2753.2003.00435.x.

36 Martin RL, Irrgang JJ. A survey of self-reported outcome instruments for the foot and ankle. J Orthop Sports Phys Ther 2007;37:72-84. doi:10.2519/jospt.2007.2403

37 Sanders R, Fortin P, DiPasquale T, Walling A. Operative treatment in 120 displaced intraarticular calcaneal fractures. Results using a prognostic computed tomography scan classification. Clin Orthop Relat Res 1993;(290):87-95

38 Karlsson J, Peterson L. Evaluation of ankle joint function: The use of a scoring scale. Foot 1991;1:15-9doi:10.1016/0958-2592(91)90006-W.

39 Braun BL. Effects of ankle sprain in a general clinic population 6 to 18 months after medical evaluation. Arch Fam Med 1999;8:143-8. doi:10.1001/archfami.8.2.143.

40 van Rijn RM, van Os AG, Bernsen RM, Luijsterburg PA, Koes BW, Bierma-Zeinstra SM. What is the clinical course of acute ankle sprains? A systematic literature review. Am J Med 2008;121:324-331. e6. doi:10.1016/j.amjmed.2007.11.018.
41 Petersen EJ, Irish SM, Lyons CL, et al. Reliability of water volumetry and the figure of eight method on subjects with ankle joint swelling. J Orthop Sports Phys Ther 1999;29:609-15. doi:10.2519| jospt.1999.29.10.609.

42 Bennell KL, Talbot RC, Wajswelner H, Techovanich W, Kelly DH, Hall AJ. Intra-rater and inter-rater reliability of a weight-bearing lunge measure of ankle dorsiflexion. Aust J Physiother 1998;44:175-80. doi:10.1016/S0004-9514(14)60377-9.

43 Novak AC, Brouwer B. Strength and aerobic requirements during stai ambulation in persons with chronic stroke and healthy adults. Arch Phys Med Rehabil 2012;93:683-9. doi:10.1016/j.apmr.2011.10.009.

44 Littell RC, Milliken GA, Stroup WW, Wolfinger RD, Schabenberger O. SAS for Mixed Models. SAS Institute Inc, 2006.

45 Liu M, Wei L, Zhang J. Review of guidelines and literature for handling missing data in longitudinal clinical trials with a case study. Pharm Stat 2006;5:7-18. doi:10.1002/pst.189.

46 Peters SAE, Bots ML, den Ruijter HM, et al. METEOR study group. Multiple imputation of missing repeated outcome measurements did not add to linear mixed-effects models. J Clin Epidemiol 2012;65:68695. doi:10.1016/j.jclinepi.2011.11.012.

47 Littell RC, Pendergast J, Natarajan R. Modelling covariance structure in the analysis of repeated measures data. Stat Med 2000;19:1793-819. doi:10.1002/1097-0258(20000715)19:13<1793::AIDSIM482>3.0.CO 2-0

48 Roos EM, Lohmander LS. The Knee injury and Osteoarthritis Outcome Score (KOOS): from joint injury to osteoarthritis. Health Qual Life Outcomes 2003;1:64. doi:10.1186/1477-7525-1-64

49 Schulz KF, Altman DG, Moher D. CONSORT Group. CONSORT 2010 Statement: updated guidelines for reporting parallel group randomised trials. BMC Med 2010:8:18 doi:10.1186/1741-7015-8-18.

50 Thabane L, Mbuagbaw L, Zhang S, et al. A tutorial on sensitivity analyses in clinical trials: the what, why, when and how. BMC Med Res Methodol 2013;13:92. doi:10.1186/1471-2288-13-92.

51 van de Mortel TF. Faking it: Social desirability response bias in self-report research. Aust J Adv Nurs 2008;25:40-8

52 de Bie RA, Hendriks HJM, Lenssen AF, et al. Clinical practice guidelines for physiotherapy in patients with acute ankle sprain [with systematic review]. http://www.pedro.fhs.usyd.edu.au/index.html

53 van der Wees PV, Lenssen AF, Feijts YAEJ, et al. KNGF guideline for physical therapy in patients with acute ankle sprain - practice guidelines. Suppl Dutch J Phys Ther 2006;116(Suppl 5):1-30

54 Shultz S, Olszewski A, Ramsey O, Schmitz M, Wyatt V, Cook C. A systematic review of outcome tools used to measure lower leg conditions. Int / Sports Phys Ther 2013;8:838-48.

55 Greenland S, Rothman KJ. Fundamentals of Epidemiological Data Analysis. In: Rothman KJ, Greenland S, Lash TL, eds. Modern epidemiology.Lippincott Williams \& Wilkins, 2008: 216

56 Konradsen L, Bech L, Ehrenbjerg M, Nickelsen T. Seven years follow-up after ankle inversion trauma. Scand J Med Sci Sports 2002;12:129-35. doi:10.1034/j.1600-0838.2002.02104.x.

57 Thomeé R. A comprehensive treatment approach for patellofemoral pain syndrome in young women. Phys Ther 1997;77:1690-703. 